\title{
Electromagnetic Dissociation of Au Targets by Relativistic Pb Projectiles
}

\author{
J.C. Hill ${ }^{\text {a }}$ A. Petridis ${ }^{\text {a }}$ B. Fadem ${ }^{\text {a }}$ F.K. Wohn ${ }^{\text {a }}$ \\ ${ }^{a}$ Iowa State University, Ames, Iowa 50011
}

\begin{abstract}
Electromagnetic Dissociation (ED) occurs in collisions of relativistic heavy ions involving impact parameters larger than the nuclear interaction radius. In such collisions strong electromagnetic fields acting at the nucleus can produce, for high charges and ultrarelativistic energies, cross sections much larger than the total nuclear interaction cross section. In ED collisions absorption of a virtual photon generally leads to excitation of a nuclear giant resonance. The NA53 experiment studied ED by bombarding Au targets with $158 \mathrm{GeV} /$ nucleon $\mathrm{Pb}$ projectiles from the SPS accelerator. Preliminary values of $\sigma_{E D}$ for the one- and two-neutron removal processes were determined to be $26.4 \pm 4.0$ and $4.6 \pm 0.7$ barns, respectively. Theoretical predictions for $\sigma_{E D}$ were calculated including the effects of both the E1 and E2 giant resonances. The calculations are extended to energies planned for heavy ion collisions at the RHIC and LHC colliders.
\end{abstract}

\section{Introduction}

Relativistic Heavy Ion (RHI) physics emphasizes the study of hadronic interactions at small impact parameters but at the energies planned for the RHIC and LHC colliders most of the reaction cross section for heavy nuclei will result from non-hadronic processes due to electromagnetic interactions. Such a purely electromagnetic process is Electromagnetic Dissociation (ED) which occurs in RHI collisions involving impact parameters larger than the strong interaction radius. Strong electromagnetic fields incident on the nucleus can result in the absorption of a virtual photon by the nucleus which usually results in the excitation of a giant multipole resonance. Deexcitation occurs primarily by means of neutron emission since at giant resonance energies charged particle emission is retarded by the Coulomb barrier. ED was first observed by Heckman and Lindstrom[1] in projectile fragmentation and in fragmentation of $\mathrm{Au}$ and Co targets by our group[2]. We report here the initial results from experiment NA53 at CERN. Preliminary values for the one- 


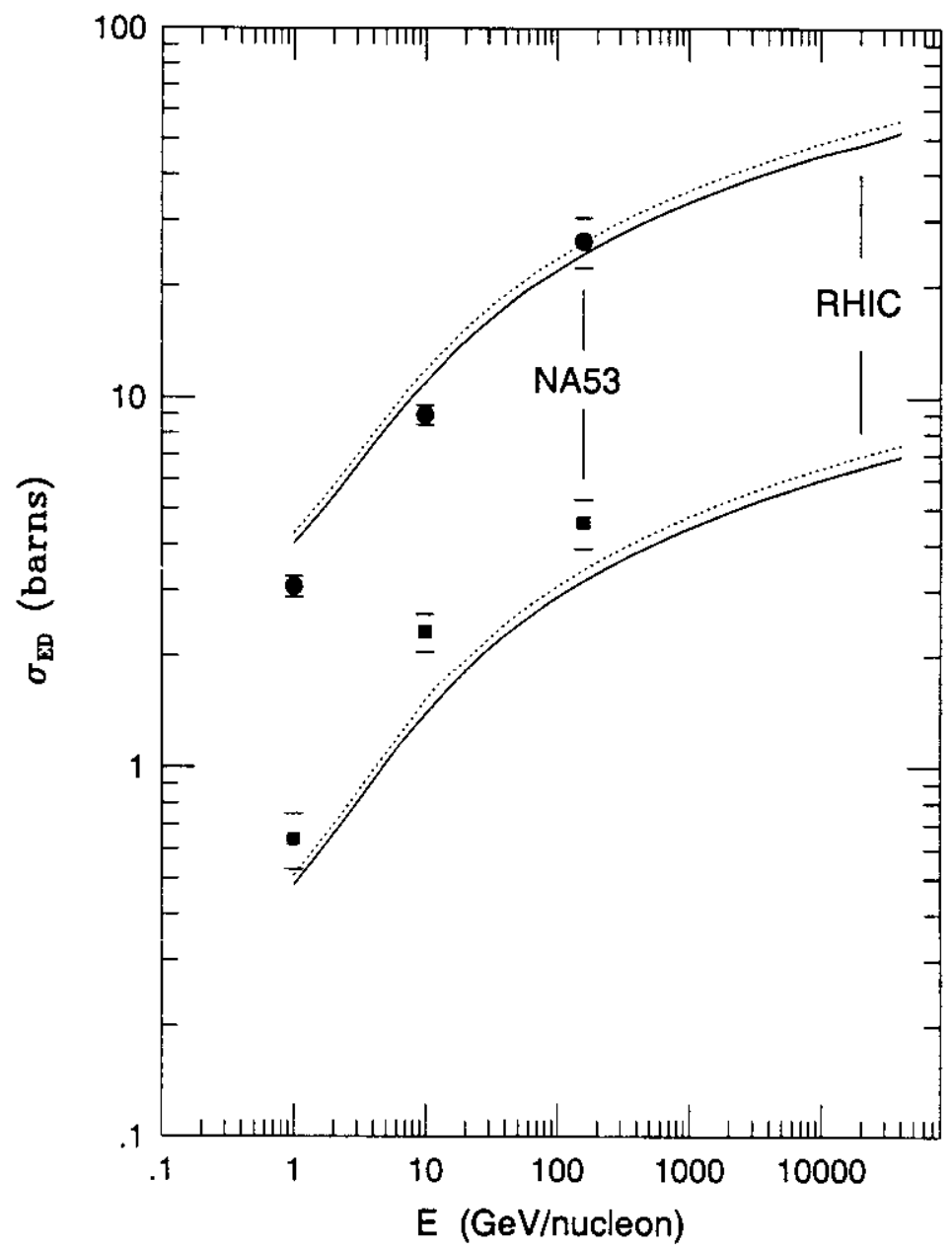

Fig. 1. Calculated $\sigma_{E D}$ for Au targets. The upper and lower curves are for one- and two-neutron removal reactions, respectively. and the solid and dashed curves are for $\mathrm{Au}$ and $\mathrm{Pb}$ projectiles, respectively.

contributions are for the E1 and E2 (isoscalar and isovector) resonances. The corrections are made using the method of Norbury[8]. The resonance energies and widths used were 10.8 and $2.9 \mathrm{MeV}$ for the isoscalar and 23.0 and 7.0 $\mathrm{MeV}$ for the isovector E2 resonances, respectively[9]. A sum rule strength of $95 \%$ was used. The prescription for the minimum impact parameter used was that of Benesh et al.[10]. In Figure 1 the measured $\sigma_{E D}$ for one- and twoneutron removal from $\mathrm{Au}$ targets by $\mathrm{Pb}$ beams from the SPS measured in this work are compared with the calculations described above. Also shown for comparison are measurements of $1 \mathrm{GeV} /$ nucleon $\mathrm{Au}$ projectiles on $\mathrm{Au}$ targets by Aumann et al.[11] and $9.9 \mathrm{GeV} /$ nucleon Au projectiles on Au targets by Hill et al.[12]. Although agreement is reasonable the theoretical predictions are systematically too low or high for the one- and two-neutron removal processes, respectively. Llope and Braun-Munzinger[13] suggested that it is possible to 
excite a multiphonon giant E1 resonance by absorption of several photons and this effect has been used to partially explain[11] the enhancement of the twoneutron removal process relative to one-neutron removal with $1 \mathrm{GeV} /$ nucleon $\mathrm{Au}$ beams on $\mathrm{Au}$ targets.

\section{Extrapolation to Collider Energies}

Due to the large values of $\sigma_{E D}$ at ultrarelativistic energies ED will be a major determinant of the quality and lifetime of stored beams at the RHIC and LHC heavy ion colliders. The results for both one- and two-neutron removal ED reactions for $\mathrm{Au}+\mathrm{Au}$ at RHIC are shown on Figure 1. The calculated values are 49 and 7 barns, respectively. The $x$ axis of the figure shows the equivalent energy for a projectile incident on a stationary target. For the beams expected for $\mathrm{Pb}+\mathrm{Pb}$ collisions at the LHC the corresponding $\sigma_{E D} \mathrm{~s}$ are about 92 and 17 barns, respectively.

\section{Acknowledgements}

We wish to thank the members of the NA50 collaboration for their hospitality and help during data taking with the $\mathrm{Pb}$ beam. This work was supported by a grant from the Nuclear Physics Division of the U.S. Department of Energy.

\section{References}

[1] H.H. Heckman and P.J. Lindstrom, Phys. Rev. Lett. 37 (1976) 56

[2] M.T. Mercier et al., Phys. Rev., C33 (1986) 1655

[3] J.C. Hill et al., Phys. Rev., C38 (1988) 1722

[4] D.L. Olson et al., Phys. Rev., C28 (1983) 1602

[5] E.J. Williams, Proc. Roy. Soc., A139 (1933) 163

[6] A. Veyssiere et al., Nucl. Phys., A159 (1970) 561

[7] B.L. Berman et al., Phys. Rev., C36 (1987) 1286

[8] J.W. Norbury, Phys. Rev., C41 (1990) 372

[9] F.E. Bertrand, Annu. Rev. Nucl. Sci., 26 (1976) 457

[10] C.J. Benesh et al., Phys. Rev., C40 (1989) 1198

[11] T. Aumann et al., Phys. Rev., C47 (1993) 1728

[12] J.C. Hill et al., Proceedings of HIPAGS 96, p 286, edited by C.A. Pruneau et al., Detroit, MI, August 1996

[13] W.J. Llope and P. Braun-Munzinger, Phys. Rev., C41 (1990) 2644 
and two-neutron removal $\mathrm{ED}$ cross sections from $\mathrm{Au}$ targets are given. $\mathrm{Be}-$ low we describe the experiment and calculations leading to the measured ED cross sections. The results are compared with calculations using a WeizsackerWilliams virtual-photon spectrum taking into account the effects of both the $\mathrm{E} 1$ and E2 giant resonances. The calculations are extrapolated to energies planned for the RHIC and LHC colliders.

\section{Experimental Method and Results}

$\mathrm{Au}$ targets of $0.005 \mathrm{~mm}$ thickness were bombarded with $158 \mathrm{GeV} /$ nucleon ${ }^{208} \mathrm{~Pb}$ projectiles from the SPS accelerator. Beam intensities were typically $4 \times 10^{7}$ per spill and the total fluence was $3.4 \times 10^{10}$ projectiles on the target. The beam intensity was determined by counting signals from the NA50 segmented beam hodoscope. The saturation activities for nuclides of interest were measured by counting $\gamma$ rays for periods of up to several months. The intensities of the 356- and $99-\mathrm{keV} \gamma$ transitions were measured in order to determine the saturation activities of ${ }^{196} A u$ and ${ }^{195} A u$, respectively. Total cross sections were then calculated for one- and two-neutron removal. A number of corrections described earlier[3] are in the process of being accurately determined, therefore the cross sections reported here are preliminary. In order to separate out the nuclear contribution to the measured cross section the concept of factorization[4] was used. We assume that the yield of a fragment from the target due to nuclear interactions is independent of the beam except through a constant geometric factor that is the same for all fragments. Processes containing significant ED contributions strongly violate factorization. The estimate of the nuclear contribution has a large percentage error but at SPS energies makes only a small contribution to the error of the ED cross section. The preliminary measured results for $\sigma_{t o t}, \sigma_{n u c}$ and $\sigma_{E D}$ are given in the Table 1 below.

Table 1

Cross sections for $\mathrm{Pb}$ beams on $\mathrm{Au}$ targets.

\begin{tabular}{cccc}
\hline Nuclide & $\sigma_{\text {tot }}(b)$ & $\sigma_{\text {nuc }}(b)$ & $\sigma_{E D}(b)$ \\
\hline${ }^{196} \mathrm{Au}$ & $26.7 \pm 4.0$ & $0.3 \pm 0.1$ & $26.4 \pm 4.0$ \\
${ }^{195} \mathrm{Au}$ & $4.7 \pm 0.7$ & $0.13 \pm 0.04$ & $4.6 \pm 0.7$ \\
\hline
\end{tabular}

\section{Comparison with Theory}

The $\sigma_{E D}$ was calculated by integrating over the product of equivalent photon number $N_{\gamma}$ obtained using the method of virtual quanta[5] and the photonuclear cross section $\sigma_{\gamma}$ of Veyssiere et al.[6] multiplied by a factor of 0.93[7]. At ultrarelativistic energies $N_{\gamma}$ is the same for different multipolarities but this approximation is not valid at intermediate energies. The only significant 